\title{
Kinematic quantities and Raychaudhuri equations in a 5D universe
}

\author{
Aurel Bejancu ${ }^{\mathrm{a}}$ \\ Department of Mathematics, Kuwait University, P.O. Box 5969, 13060 Safat, Kuwait
}

Received: 6 June 2015 / Accepted: 9 July 2015 / Published online: 28 July 2015

(C) The Author(s) 2015. This article is published with open access at Springerlink.com

\begin{abstract}
Based on some ideas that have emerged from the classical Kaluza-Klein theory, we present a 5D universe as a product bundle over the 4D spacetime. This enables us to introduce and study two categories of kinematic quantities (expansions, shear, vorticity) in a 5D universe. One category is related to the fourth dimension (time), and the other one comes from the assumption of the existence of the fifth dimension. The Raychaudhuri type equations that we obtain in the paper, lead us to results on the evolution of both the $4 \mathrm{D}$ expansion and the $5 \mathrm{D}$ expansion in a 5D universe.
\end{abstract}

\section{Introduction}

As well known, the $(1+3)$ threading of a $4 \mathrm{D}$ spacetime was developed in order to relate physics and geometry to the observations. This theory is based on the existence of a congruence of timelike curves and it was successfully applied to relativistic cosmology [1], the study of gravitoelectromagnetism [2-4], the splitting of Einstein equations [5-7], and to some other physical theories.

In the present paper, we extend the above theory to a $(1+1+3)$ threading of a 5D universe. As far as we know, there are two important five-dimensional gravity theories: the brane-world gravity $[8,9]$, and the space-time-matter theory $[10,11]$. In the theory we develop in the present paper, the 4D spacetime $M$ is the base manifold of a submersion on the 5D universe $\bar{M}$, while in the above mentioned theories, $M$ is an embedded submanifold of $\bar{M}$. The new geometric configuration of $\bar{M}$ enables us to consider two orthogonal line bundles: the temporal distribution $\mathcal{T} \bar{M}$ which defines a congruence of timelike curves, and the vertical distribution $\mathcal{V} \bar{M}$ which is tangent to a congruence of spacelike curves. The spatial distribution $\mathcal{S} \bar{M}$ is the complementary orthogonal distribution to $\mathcal{T} \bar{M} \oplus \mathcal{V} \bar{M}$ in the tangent bundle of $\bar{M}$. The kinematic quantities (expansion, shear, vorticity) in $\bar{M}$,

a e-mail: aurel.bejancu@ku.edu.kw are introduced as spatial tensor fields on $\bar{M}$, which roughly speaking, behave like tensor fields on a three-dimensional manifold. In order to define a covariant derivative of such tensor fields with respect to any vector field on $\bar{M}$, we introduce the Riemannian spatial connection, which is a metric linear connection on $\mathcal{S} \bar{M}$. Finally, we obtain three Raychaudhuri type equations, which enable us to study the evolution of the expansion in the 5D universe $\bar{M}$.

Now, we outline the content of the paper. In Sect. 2 we present the geometric structure of the 5D universe $(\bar{M}, \bar{g})$ [see (2.12)], and we construct the adapted frame and coframe fields $\left\{\delta / \delta x^{0}, \delta / \delta x^{\alpha}, \partial / \partial x^{4}\right\}$ and $\left\{\delta x^{0}, \mathrm{~d} x^{\alpha}, \delta x^{4}\right\}$, respectively. Then, in Sect. 3 we consider the $4 \mathrm{D}$ velocity $\xi=\delta / \delta x^{0}$ and the $5 \mathrm{D}$ velocity $\eta=\partial / \partial x^{4}$, and obtain the expression of the line element of $\bar{g}$ with respect to the adapted coframe field [cf.(3.4)]. The 4D and 5D kinematic quantities: expansion, shear, and vorticity tensor fields are defined in Sect. 4 . They are geometric objects on $\bar{M}$, whose local components with respect to adapted frame and coframe fields behave as tensor fields on a three-dimensional manifold. The Riemannian spatial connection, which we introduce in Sect. 5, has an important role in the study. Here, we show that the LeviCivita connection of $(\bar{M}, \bar{g})$ is completely determined by the kinematic quantities, the Riemannian spatial connection, and the spatial tensor fields we introduced in Sect. 4 [cf. (5.12)]. In Sect. 6 we show that each spatial tensor field defines a tensor field of the same type on $\bar{M}$, and evaluate the covariant derivatives of both velocities [cf. (6.9), (6.10)]. In Sect. 7 we derive the Raychaudhuri equations with respect to the expansions $\Theta$ and $K$ of the 5D universe [cf. (7.14), (7.16), (7.22)]. In spite of the full generality approached in the paper, we can see that due to the spatial tensor fields and to the Riemannian spatial connection, the calculations can easily be handled. By using the Raychaudhuri equations we state two results on the evolution of $\Theta$ and $K$ in $(\bar{M}, \bar{g})$ (cf. Theorems 7.1, 7.2). The conclusions on the theory we develop in the paper are presented in Sect. 8. 


\section{Adapted frames and coframes in a 5D universe}

Let $M$ and $K$ be manifolds of dimension four and one, respectively, and $\bar{M}=M \times K$ be the product bundle over $M$ with fiber $K$. Then a coordinate system $\left(x^{i}\right)$ on $M$ determines a coordinate system $\left(x^{a}\right)=\left(x^{i}, x^{4}\right)$ on $\bar{M}$, where $x^{4}$ is the fiber coordinate. A general Kaluza-Klein theory on $\bar{M}$ is developed with respect to the gauge group $U(1)$, and the coordinate transformations on $\bar{M}$ are given by

(a) $\tilde{x}^{i}=\tilde{x}^{i}\left(x^{0}, x^{1}, x^{2}, x^{3}\right)$;

(b) $\tilde{x}^{4}=x^{4}+\bar{f}\left(x^{0}, x^{1}, x^{2}, x^{3}\right)$.

Note that the above coordinate transformations are induced by the special geometric structure of $\bar{M}$, according to a general Kaluza-Klein theory. The space-time-matter theory (cf. $[10,11])$ is developed in a more general setting of coordinate transformations. As a consequence of (2.1) we deduce that the natural frame fields $\partial / \partial x^{a}$ and $\partial / \partial \tilde{x}^{a}$ are related by
(a) $\frac{\partial}{\partial x^{i}}=\frac{\partial \widetilde{x}^{k}}{\partial x^{i}} \frac{\partial}{\partial \widetilde{x}^{k}}+\frac{\partial \bar{f}}{\partial x^{i}} \frac{\partial}{\partial \widetilde{x}^{4}}$,
(b) $\frac{\partial}{\partial x^{4}}=\frac{\partial}{\partial \widetilde{x}^{4}}$.

Throughout the paper we use the ranges of indices: $a, b, c, \ldots$ $\in\{0,1,2,3,4\}, i, j, k, \ldots \in\{0,1,2,3\}$, and $\alpha, \beta, \gamma, \ldots \in$ $\{1,2,3\}$. Also, for any vector bundle $E$ over $\bar{M}$ denote by $\Gamma(E)$ the $\mathcal{F}(\bar{M})$-module of smooth sections of $E$, where $\mathcal{F}(\bar{M})$ is the algebra of smooth functions on $\bar{M}$.

From (2.2(b)) we see that there exists a globally defined vector field $\eta$ on $\bar{M}$, which is given locally by $\partial / \partial x^{4}$. Then denote by $\mathcal{V} \bar{M}$ the line bundle over $\bar{M}$ spanned by $\eta$, and call it the vertical distribution on $\bar{M}$. Suppose that $\bar{M}$ is endowed with a Lorentz metric $\bar{g}$ such that

$\bar{g}\left(\frac{\partial}{\partial x^{4}}, \frac{\partial}{\partial x^{4}}\right)=\Psi^{2}$

where $\Psi$ is a non-zero function that is globally defined on $\bar{M}$. Denote by $\mathcal{H} \bar{M}$ the complementary orthogonal vector bundle to $\mathcal{V} \bar{M}$ in the tangent bundle $T \bar{M}$ of $\bar{M}$, and call it the horizontal distribution on $\bar{M}$. Hence we have the Whitney decomposition

$T \bar{M}=\mathcal{H} \bar{M} \oplus \mathcal{V} \bar{M}$

Now, suppose that on $M$ there exists a globally defined vector field $U$, which induces a special coordinate system $\left(x^{i}\right)$ on $M$ such that $U=\partial / \partial x^{0}$. Two such coordinate systems $\left(x^{i}\right)$ and $\left(\widetilde{x}^{i}\right)$ on $M$ are related by

(a) $\tilde{x}^{\alpha}=\tilde{x}^{\alpha}\left(x^{1}, x^{2}, x^{3}\right)$;

(b) $\tilde{x}^{0}=x^{0}+f\left(x^{1}, x^{2}, x^{3}\right)$.
Thus, the coordinate transformations on $\bar{M}$ are given by (2.1(b)) and (2.5). Then (2.2(a)) becomes

(a) $\frac{\partial}{\partial x^{\alpha}}=\frac{\partial \widetilde{x}^{\gamma}}{\partial x^{\alpha}} \frac{\partial}{\partial \widetilde{x}^{\gamma}}+\frac{\partial f}{\partial x^{\alpha}} \frac{\partial}{\partial \widetilde{x}^{0}}+\frac{\partial \bar{f}}{\partial x^{\alpha}} \frac{\partial}{\partial \widetilde{x}^{4}}$,

(b) $\frac{\partial}{\partial x^{0}}=\frac{\partial}{\partial \widetilde{x}^{0}}+\frac{\partial \bar{f}}{\partial x^{0}} \frac{\partial}{\partial \widetilde{x}^{4}}$,

where $\left\{\partial / \partial x^{i}\right\}$ is the lift of the natural frame field on $M$ to $\bar{M}$.

Next, suppose that the lift of $\partial / \partial x^{0}$ to $\bar{M}$ is timelike with respect to $\bar{g}$ and denote by $\delta / \delta x^{0}$ its projection on $\mathcal{H} \bar{M}$ with respect to (2.4). Thus there exists locally on $\bar{M}$ a unique function $A_{0}$, such that

$\frac{\delta}{\delta x^{0}}=\frac{\partial}{\partial x^{0}}-A_{0} \frac{\partial}{\partial x^{4}}$

By direct calculations, using (2.2(b)), (2.6(b)), and (2.7), we deduce that
(a) $\frac{\delta}{\delta x^{0}}=\frac{\delta}{\delta \tilde{x}^{0}}$,
(b) $A_{0}=\tilde{A}_{0}+\frac{\partial \bar{f}}{\partial x^{0}}$,

with respect to the coordinate transformations on $\bar{M}$. From (2.8(a)) we conclude that there exists a globally defined horizontal vector field $\xi$ on $\bar{M}$, which is locally given by $\delta / \delta x^{0}$. Moreover, $\xi$ is a timelike vector field on $\bar{M}$. To show this, we consider the line element of $\bar{g}$ given by
(a) $\mathrm{d} \bar{s}^{2}=\bar{g}_{a b} \mathrm{~d} x^{a} \mathrm{~d} x^{b}$,
(b) $\bar{g}_{a b}=g\left(\frac{\partial}{\partial x^{a}}, \frac{\partial}{\partial x^{b}}\right)$.

Then, taking into account that $\xi$ is orthogonal to $\eta$, and using (2.3), (2.7) and (2.9(b)), we obtain

$A_{0}=\Psi^{-2} \bar{g}_{04}$

By similar calculations we infer that

$\bar{g}\left(\frac{\delta}{\delta x^{0}}, \frac{\delta}{\delta x^{0}}\right)=\bar{g}_{00}-\left(A_{0} \Psi\right)^{2}$.

As $\bar{g}_{00}<0$, there exists a globally defined non-zero function $\Phi$ on $\bar{M}$ such that

$\bar{g}\left(\frac{\delta}{\delta x^{0}}, \frac{\delta}{\delta x^{0}}\right)=-\Phi^{2}$

and therefore $\xi$ is timelike. Denote by $\mathcal{T} \bar{M}$ the line bundle spanned by $\xi$ and call it the temporal distribution on $\bar{M}$. Thus the decomposition from (2.4) admits the refinement

$T \bar{M}=\mathcal{T} \bar{M} \oplus \mathcal{S} \bar{M} \oplus \mathcal{V} \bar{M}$

where $\mathcal{S} \bar{M}$ is the complementary orthogonal bundle to $\mathcal{T} \bar{M}$ in $\mathcal{H} \bar{M}$. As $\bar{g}$ is a Lorentz metric, and $\mathcal{T} \bar{M}$ is a timelike 
vector bundle, we conclude that $\mathcal{S} \bar{M}$ is spacelike. Thus, we are entitled to call $\mathcal{S} \bar{M}$ the spatial distribution on $\bar{M}$.

Next, consider $\left\{\partial / \partial x^{\alpha}\right\}, \alpha \in\{1,2,3\}$, as vector fields locally defined on $\bar{M}$ and denote by $\left\{\delta / \delta x^{\alpha}\right\}$ their projections on $\mathcal{S} \bar{M}$ with respect to the decomposition (2.12). Thus, we have

$\frac{\delta}{\delta x^{\alpha}}=\frac{\partial}{\partial x^{\alpha}}-B_{\alpha} \frac{\delta}{\delta x^{0}}-A_{\alpha} \frac{\partial}{\partial x^{4}}$,

where $A_{\alpha}$ and $B_{\alpha}$ are locally defined functions on $\bar{M}$. By direct calculations, using (2.2(b)), (2.6(a)), (2.7), and (2.8(a)) in (2.13), we deduce that
(a) $\frac{\delta}{\delta x^{\alpha}}=\frac{\partial \tilde{x}^{\gamma}}{\partial x^{\alpha}} \frac{\delta}{\delta \tilde{x}^{\gamma}}$
(b) $B_{\alpha}=\frac{\partial \tilde{x}^{\gamma}}{\partial x^{\alpha}} \tilde{B}_{\gamma}+\frac{\partial f}{\partial x^{\alpha}}$,
(c) $A_{\alpha}=\frac{\partial \tilde{x}^{\gamma}}{\partial x^{\alpha}} \tilde{A}_{\gamma}+\frac{\partial f}{\partial x^{\alpha}} \tilde{A}_{0}+\frac{\partial \bar{f}}{\partial x^{\alpha}}$.

By the above geometric construction, we introduce the orthogonal frame field $\left\{\delta / \delta x^{0}, \delta / \delta x^{\alpha}, \partial / \partial x^{4}\right\}$, which we call an adapted frame field on $\bar{M}$. Its dual frame field $\left\{\delta x^{0}, \mathrm{~d} x^{\alpha}, \delta x^{4}\right\}$, where we put
(a) $\delta x^{0}=\mathrm{d} x^{0}+B_{\alpha} \mathrm{d} x^{\alpha}$,
(b) $\delta x^{4}=\mathrm{d} x^{4}+A_{i} \mathrm{~d} x^{i}$,

is called an adapted coframe field on $\bar{M}$.

The pair $(\bar{M}, \bar{g})$ with the geometric configuration described in this section is called a 5D universe and is going to be the main object studied in the present paper. It is important to note that the $5 \mathrm{D}$ universe that we introduce in this paper is different from the ones considered in the well-known theories: braneworld theory $[8,9]$ and space-time-matter theory $[10,11]$. This is because in the present theory the $4 \mathrm{D}$ spacetime is the base manifold of a submersion defined on the 5D universe, while in the above theories the 4D spacetime is considered embedded in the 5D universe.

\section{4D and 5D velocities in a 5D universe}

Let $(\bar{M}, \bar{g})$ be a 5D universe with the line element given by (2.9). From the previous section we conclude that $\bar{M}$ admits a double threading by two orthogonal congruences of curves. These congruences are defined by the timelike vector field $\xi$ and by the spacelike vector field $\eta$, which we call the 4D velocity and $5 \mathrm{D}$ velocity, respectively. The name $4 \mathrm{D}$ velocity for $\xi$ is justified by the fact that its integral curves are tangent on their entire length to the horizontal distribution, whose fibers are four-dimensional. On the contrary, the integral curves of $\eta$ are orthogonal to $\mathcal{H} \bar{M}$, and therefore they are intimately related to the fifth dimension. Moreover, by using (2.7), we obtain
(a) $\left[\frac{\delta}{\delta x^{0}}, \frac{\partial}{\partial x^{4}}\right]=a_{0} \frac{\partial}{\partial x^{4}}$,
(b) $a_{0}=\frac{\partial A_{0}}{\partial x^{4}}$.

Thus, the distribution $\mathcal{T} \bar{M} \oplus \mathcal{V} \bar{M}$ is an integrable distribution, and therefore, the $5 \mathrm{D}$ universe admits also a foliation by surfaces, whose transversal bundle $([12$, p. 7$])$ is the spatial distribution $\mathcal{S} \bar{M}$. Next, denote by $h$ the Riemannian metric induced by $\bar{g}$ on $\mathcal{S} \bar{M}$, and put

$$
\begin{aligned}
h_{\alpha \beta} & =h\left(\frac{\delta}{\delta x^{\beta}}, \frac{\delta}{\delta x^{\alpha}}\right) \\
& =\bar{g}\left(\frac{\delta}{\delta x^{\beta}}, \frac{\delta}{\delta x^{\alpha}}\right), \quad \alpha, \beta \in\{1,2,3\} .
\end{aligned}
$$

Then, by using (2.3), (2.9(b)), (2.11), (2.13), and (3.2), we deduce that

$h_{\alpha \beta}=\bar{g}_{\alpha \beta}+\Phi^{2} B_{\alpha} B_{\beta}-\Psi^{2} A_{\alpha} A_{\beta}$.

Due to (2.3), (2.11) and (3.3), the line element with respect to the adapted coframe field has the simple form

$\mathrm{d} \bar{s}^{2}=-\Phi^{2}\left(\delta x^{0}\right)^{2}+h_{\alpha \beta} \mathrm{d} x^{\alpha} \mathrm{d} x^{\beta}+\Psi^{2}\left(\delta x^{4}\right)^{2}$.

Now, in order to find the covariant local components of the above velocities we consider the 1 -forms $\xi^{\star}$ and $\eta^{\star}$ given by
(a) $\xi^{\star}(X)=\bar{g}(X, \xi)$,
(b) $\eta^{\star}(X)=\bar{g}(X, \eta), \quad \forall X \in \Gamma(T \bar{M})$.

Then we put
(a) $\xi_{a}=\xi^{\star}\left(\frac{\partial}{\partial x^{a}}\right)$
(b) $\eta_{a}=\eta^{\star}\left(\frac{\partial}{\partial x^{a}}\right), \quad a \in\{0,1,2,3,4\}$,

and by using (2.3), (2.7), (2.11), (2.13), (3.5), and (3.6), we obtain
(a) $\xi_{0}=-\Phi^{2}$,
(b) $\xi_{\alpha}=-\Phi^{2} B_{\alpha}, \quad$ (c) $\xi_{4}=0, \quad \forall \alpha \in\{1,2,3\}$,

and
(a) $\eta_{i}=\Psi^{2} A_{i}$,
(b) $\eta_{4}=\Psi^{2}, \quad \forall i \in\{0,1,2,3\}$.

By using (3.8(a)) and (3.7(b)) in (2.7) and (2.13), we infer that
(a) $\frac{\delta}{\delta x^{0}}=\frac{\partial}{\partial x^{0}}-\Psi^{-2} \eta_{0} \frac{\partial}{\partial x^{4}}$,
(b) $\frac{\delta}{\delta x^{\alpha}}=\frac{\partial}{\partial x^{\alpha}}+\Phi^{-2} \xi_{\alpha} \frac{\delta}{\delta x^{0}}-\Psi^{-2} \eta_{\alpha} \frac{\partial}{\partial x^{4}}$.

Similarly, (2.15) becomes

(a) $\delta x^{0}=d x^{0}-\Phi^{-2} \xi_{\alpha} \mathrm{d} x^{\alpha}$,

(b) $\delta x^{4}=\mathrm{d} x^{4}+\Psi^{-2} \eta_{i} \mathrm{~d} x^{i}$. 
In what follows, we also call $\xi^{\star}=\left(\xi_{a}\right)$ and $\eta^{\star}=\left(\eta_{a}\right)$ the 4D velocity and the $5 \mathrm{D}$ velocity in $(\bar{M}, \bar{g})$, respectively.

\section{Spatial tensor fields and kinematic quantities in a 5D universe}

In this section we introduce spatial tensor fields in $(\bar{M}, \bar{g})$ as geometric objects whose local components behave as the ones of tensor fields on a three-dimensional manifold. In particular, we define the expansion, shear and vorticity tensor fields as spatial tensor fields.

First, by using (2.14(a)) in (3.2), we deduce that the local components of the Riemannian metric $h$ on $\mathcal{S} \bar{M}$ satisfy

$h_{\alpha \beta}=\widetilde{h}_{\mu \nu} \frac{\partial \tilde{x}^{\mu}}{\partial x^{\alpha}} \frac{\partial \tilde{x}^{\nu}}{\partial x^{\beta}}, \quad \alpha, \beta \in\{1,2,3\}$,

with respect to the coordinate transformations on $\bar{M}$. Also, the entries of the inverse of the $3 \times 3$ matrix $\left[h_{\alpha \beta}\right]$ satisfy

$\tilde{h}^{\mu \nu}=h^{\alpha \beta} \frac{\partial \tilde{x}^{\mu}}{\partial x^{\alpha}} \frac{\partial \tilde{x}^{v}}{\partial x^{\beta}}, \quad \mu, v \in\{1,2,3\}$.

Thus, $h_{\alpha \beta}$ and $h^{\alpha \beta}$ are locally functions on the fivedimensional manifold $\bar{M}$, but they are transformed as the local components of some tensor fields of type $(0,2)$ and $(2,0)$ on a three-dimensional manifold. This leads us to an important category of geometric objects on $\bar{M}$. Namely, we say that the functions $T_{\alpha_{1} \cdots \alpha_{q}}^{\gamma_{1} \cdots \gamma_{p}}\left(x^{a}\right)$ define a spatial tensor field of type $(p, q)$ in the 5D universe $(\bar{M}, \bar{g})$, if they satisfy

$T_{\alpha_{1} \cdots \alpha_{q}}^{\gamma_{1} \cdots \gamma_{p}} \frac{\partial \tilde{x}^{\mu_{1}}}{\partial x^{\gamma_{1}}} \cdots \frac{\partial \tilde{x}^{\mu_{p}}}{\partial x^{\gamma_{p}}}=\tilde{T}_{\nu_{1} \cdots \nu_{q}}^{\mu_{1} \cdots \mu_{p}} \frac{\partial \tilde{x}^{\nu_{1}}}{\partial x^{\alpha_{1}}} \cdots \frac{\partial \tilde{x}^{\nu_{q}}}{\partial x^{\alpha_{q}}}$,

with respect to the coordinate transformations on $\bar{M}$. By using (4.1) and (4.2), we see that $h_{\alpha \beta}$ and $h^{\alpha \beta}$ define spatial tensor fields of types $(0,2)$ and $(2,0)$, respectively.

Next, by direct calculations using (2.7) and (2.13), we obtain

(a) $\left[\frac{\delta}{\delta x^{\alpha}}, \frac{\delta}{\delta x^{0}}\right]=b_{\alpha} \frac{\delta}{\delta x^{0}}+a_{\alpha} \frac{\partial}{\partial x^{4}}$,

(b) $\left[\frac{\delta}{\delta x^{\alpha}}, \frac{\partial}{\partial x^{4}}\right]=d_{\alpha} \frac{\delta}{\delta x^{0}}+c_{\alpha} \frac{\partial}{\partial x^{4}}$,

(c) $\left[\frac{\delta}{\delta x^{\beta}}, \frac{\delta}{\delta x^{\alpha}}\right]=2 \omega_{\alpha \beta} \frac{\delta}{\delta x^{0}}+2 \eta_{\alpha \beta} \frac{\partial}{\partial x^{4}}$,

where we put

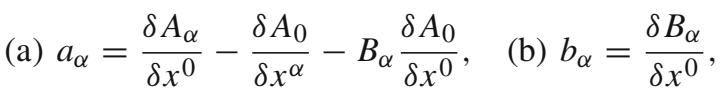

(c) $c_{\alpha}=\frac{\partial A_{\alpha}}{\partial x^{4}}-B_{\alpha} \frac{\partial A_{0}}{\partial x^{4}}, \quad$ (d) $d_{\alpha}=\frac{\partial B_{\alpha}}{\partial x^{4}}$,

(e) $\omega_{\alpha \beta}=\frac{1}{2}\left\{\frac{\delta B_{\beta}}{\delta x^{\alpha}}-\frac{\delta B_{\alpha}}{\delta x^{\beta}}\right\}$,

(f) $\eta_{\alpha \beta}=\frac{1}{2}\left\{\frac{\delta A_{\beta}}{\delta x^{\alpha}}-\frac{\delta A_{\alpha}}{\delta x^{\beta}}+B_{\alpha} \frac{\delta A_{0}}{\delta x^{\beta}}-B_{\beta} \frac{\delta A_{0}}{\delta x^{\alpha}}\right\}$.
By using (2.2(b)), (2.8(a)), and (2.14(a)) in (4.4), it is easy to check that $a_{\alpha}, b_{\alpha}, c_{\alpha}, d_{\alpha}$ define spatial tensor fields of type $(0,1)$, while $\omega_{\alpha \beta}$ and $\eta_{\alpha \beta}$ define skew-symmetric spatial tensor fields of type $(0,2)$. Moreover, from $(4.4(\mathrm{c}))$ we deduce that the spatial distribution is integrable, if and only if,

$\omega_{\alpha \beta}=0$, and $\eta_{\alpha \beta}=0, \quad \forall \alpha, \beta \in\{1,2,3\}$.

Thus, extending the terminology from $(1+3)$ threading of a 4D spacetime ([1,p. 81]), we call $\omega_{\alpha \beta}$ and $\eta_{\alpha \beta}$ the 4D vorticity tensor field and the 5D vorticity tensor field, respectively. The prefix 4D is placed in front of vorticity to emphasize that this object comes from the structure of the 4D spacetime $M$. On the contrary, the $5 \mathrm{D}$ vorticity is determined by the existence of the fifth dimension. Throughout the paper, we use this rule for some other geometric objects.

Now, we define the spatial tensor fields of type $(0,1)$ :

(a) $\phi_{\alpha}=\Phi^{-1} \frac{\delta \Phi}{\delta x^{\alpha}}, \quad$ (b) $\psi_{\alpha}=\Psi^{-1} \frac{\delta \Psi}{\delta x^{\alpha}}$,

and by using (3.7(b)) and (3.8(a)) in (4.5(e)) and (4.5(f)), we deduce that

(a) $\omega_{\alpha \beta}=\Phi^{-2}\left\{\phi_{\alpha} \xi_{\beta}-\phi_{\beta} \xi_{\alpha}+\frac{1}{2}\left(\frac{\delta \xi_{\alpha}}{\delta x^{\beta}}-\frac{\delta \xi_{\beta}}{\delta x^{\alpha}}\right)\right\}$,
(b) $\eta_{\alpha \beta}=\Psi^{-2}\left\{\eta_{\alpha} \psi_{\beta}-\eta_{\beta} \psi_{\alpha}+\frac{1}{2}\left(\frac{\delta \eta_{\beta}}{\delta x^{\alpha}}-\frac{\delta \eta_{\alpha}}{\delta x^{\beta}}\right)\right.$
$\left.+\Phi^{-2} \eta_{0}\left(\xi_{\alpha} \psi_{\beta}-\xi_{\beta} \psi_{\alpha}\right)+\frac{1}{2} \Phi^{-2}\left(\xi_{\beta} \frac{\delta \eta_{0}}{\delta x^{\alpha}}-\xi_{\alpha} \frac{\delta \eta_{0}}{\delta x^{\beta}}\right)\right\}$.

Next, we denote by $\mathcal{L}$ the Lie derivative on $\bar{M}$ and define the functions

(a) $\Theta_{\alpha \beta}=\frac{1}{2}\left(\mathcal{L}_{\frac{\delta}{\delta x^{0}}} \bar{g}\right)\left(\frac{\delta}{\delta x^{\beta}}, \frac{\delta}{\delta x^{\alpha}}\right)$,
(b) $K_{\alpha \beta}=\frac{1}{2}\left(\mathcal{L}_{\frac{\partial}{\partial x^{4}}} \bar{g}\right)\left(\frac{\delta}{\delta x^{\beta}}, \frac{\delta}{\delta x^{\alpha}}\right)$.

Then, by using (3.2), (4.4(a)), and (4.4(b)) in (4.9), we obtain

(a) $\Theta_{\alpha \beta}=\frac{1}{2} \frac{\delta h_{\alpha \beta}}{\delta x^{0}}, \quad$ (b) $K_{\alpha \beta}=\frac{1}{2} \frac{\partial h_{\alpha \beta}}{\partial x^{4}}$.

Moreover, applying $\delta / \delta x^{0}$ and $\partial / \partial x^{4}$ to (4.1), and taking into account $(2.2(\mathrm{~b})),(2.5(\mathrm{a}))$ and $(2.8(\mathrm{a}))$, we deduce that $\Theta_{\alpha \beta}$ and $K_{\alpha \beta}$ define symmetric spatial tensor fields of type $(0,2)$. We call $\Theta_{\alpha \beta}$ and $K_{\alpha \beta}$ the 4D expansion tensor field and the 5D expansion tensor field, respectively. Taking the traces of these tensor fields, we obtain the 4D expansion function $\Theta$ and the 5D expansion function $K$, given by
(a) $\Theta=\Theta_{\alpha \beta} h^{\alpha \beta}$,
(b) $K=K_{\alpha \beta} h^{\alpha \beta}$. 
Finally, we define the trace-free symmetric spatial tensor fields
(a) $\sigma_{\alpha \beta}=\Theta_{\alpha \beta}-\frac{1}{3} \Theta h_{\alpha \beta}$,
(b) $H_{\alpha \beta}=K_{\alpha \beta}-\frac{1}{3} K h_{\alpha \beta}$.

Then, inspired by the terminology from the kinematic theory in a 4D spacetime, we call $\sigma_{\alpha \beta}$ and $H_{\alpha \beta}$ the $4 \mathrm{D}$ shear tensor field and the 5D shear tensor field, respectively. As a conclusion of this section, we may say that $\left\{\omega_{\alpha \beta}, \Theta_{\alpha \beta}, \Theta, \sigma_{\alpha \beta}\right\}$ and $\left\{\eta_{\alpha \beta}, K_{\alpha \beta}, K, H_{\alpha \beta}\right\}$ are the $4 \mathrm{D}$ kinematic quantities and the 5D kinematic quantities in the 5D universe $(\bar{M}, \bar{g})$, with respect to the congruence of curves defined by $\xi$ and $\eta$, respectively.

\section{The Riemannian spatial connection in a 5D universe}

Let $(\bar{M}, \bar{g})$ be a $5 \mathrm{D}$ universe, and $\mathcal{S} \bar{M}^{\star}$ be the dual bundle of the spatial distribution $\mathcal{S} \bar{M}$. Suppose that

$$
T: \Gamma\left(\mathcal{S} \bar{M}^{\star}\right)^{p} \times \Gamma(\mathcal{S} \bar{M})^{q} \longrightarrow \mathcal{F}(\bar{M})
$$

is a $(p+q)-\mathcal{F}(\bar{M})$-multilinear mapping, and locally define the functions

$T_{\alpha_{1} \cdots \alpha_{q}}^{\gamma_{1} \cdots \gamma_{p}}=T\left(\mathrm{~d} x^{\gamma_{1}}, \ldots, \mathrm{d} x^{\gamma_{p}}, \frac{\delta}{\delta x^{\alpha_{1}}}, \ldots, \frac{\delta}{\delta x^{\alpha_{q}}}\right)$.

Then it is easy to check that $T_{\alpha_{1} \cdots \alpha_{q}}^{\gamma_{1} \cdots \gamma_{p}}$ satisfy (4.3), and therefore they define a spatial tensor field of type $(p, q)$. Conversely, suppose $T_{\alpha_{1} \cdots \alpha_{q}}^{\gamma_{1} \cdots \gamma_{p}}$ are functions satisfying (4.3). Then $T$ defined locally by (5.2) becomes an $\mathcal{F}(\bar{M})$-multilinear mapping as in (5.1).

The purpose of this section is to define covariant derivatives of the spatial tensor fields given either as in (5.1), or by their local components from (5.2). First, we consider the Levi-Civita connection $\bar{\nabla}$ on $(\bar{M}, \bar{g})$ given by (cf. [13, p. 61])

$$
\begin{aligned}
& 2 \bar{g}\left(\bar{\nabla}_{X} Y, Z\right)=X(\bar{g}(Y, Z))+Y(\bar{g}(Z, X))-Z(\bar{g}(X, Y)) \\
& \quad+\bar{g}([X, Y], Z)-\bar{g}([Y, Z], X)+\bar{g}([Z, X], Y),
\end{aligned}
$$

for all $X, Y, Z \in \Gamma(T \bar{M})$. Then define the operator

$$
\begin{aligned}
& \nabla: \Gamma(T \bar{M}) \times \Gamma(\mathcal{S} \bar{M}) \longrightarrow \Gamma(\mathcal{S} \bar{M}), \\
& \nabla(X, s Y)=\nabla_{X} S=s \bar{\nabla}_{X} S Y, \forall X, Y \in \Gamma(T \bar{M}),
\end{aligned}
$$

where $s$ is the projection morphism of $T \bar{M}$ on $\mathcal{S} \bar{M}$ with respect to (2.12). It is easy to check that $\nabla$ is a metric linear connection on $\mathcal{S} \bar{M}$, that is, we have

$\left(\nabla_{X} h\right)(s Y, s Z)=0, \quad \forall X, Y, Z \in \Gamma(T \bar{M})$,

where $h$ is the Riemannian metric on $\mathcal{S} \bar{M}$. We call $\nabla$ the Riemannian spatial connection in the 5D universe $(\bar{M}, \bar{g})$.

Taking into account that all the kinematic quantities have been defined by their local components, we need to characterize $\nabla$ by its local coefficients with respect to an adapted frame field. First, we put

$$
\begin{gathered}
\text { (a) } \nabla_{\frac{\delta}{\delta x^{\beta}}} \frac{\delta}{\delta x^{\alpha}}=\Gamma_{\alpha}^{\gamma} \frac{\delta}{\delta x^{\gamma}}, \quad \text { (b) } \nabla_{\frac{\partial}{\partial x^{0}}} \frac{\delta}{\delta x^{\alpha}}=\Gamma_{\alpha}^{\gamma} \frac{\delta}{\delta x^{\gamma}} \\
\text { (c) } \nabla_{\frac{\partial}{\partial x^{4}} \frac{\delta}{\delta x^{\alpha}}}=\Gamma_{\alpha}^{\gamma} \frac{\delta}{\delta x^{\gamma}} .
\end{gathered}
$$

Then take $X=\delta / \delta x^{\beta}, Y=\delta / \delta x^{\alpha}$, and $Z=\delta / \delta x^{\mu}$ in (5.3), and by using (3.2), (4.4(c)), (5.4), and (5.6(a)), we obtain

$\Gamma_{\alpha \beta}^{\gamma}=\frac{1}{2} h^{\gamma \mu}\left\{\frac{\delta h_{\mu \alpha}}{\delta x^{\beta}}+\frac{\delta h_{\mu \beta}}{\delta x^{\alpha}}-\frac{\delta h_{\alpha \beta}}{\delta x^{\mu}}\right\}$.

Note that formally $\Gamma_{\alpha \beta}^{\gamma}$ look like the Christoffel symbols for a Levi-Civita connection on a three-dimensional manifold, but two main differences should be pointed out:

(i) In general, $h_{\alpha \beta}$ are functions of all five variables $\left(x^{a}\right)$,

(ii) the usual partial derivatives are replaced here by the operators defined by (2.13).

Throughout the paper, we use $h_{\alpha \beta}$ and $h^{\alpha \beta}$ for lowering and raising Greek indices. As an example, for the vorticity tensor fields we have
(a) $\omega_{\beta}^{\gamma}=h^{\gamma \alpha} \omega_{\alpha \beta}$,
(c) $\eta_{\beta}^{\gamma}=h^{\gamma \alpha} \eta_{\alpha \beta}$,
(b) $\omega^{\gamma \mu}=h^{\gamma \alpha} h^{\mu \beta} \omega_{\alpha \beta}$,
(d) $\eta^{\gamma \mu}=h^{\gamma \alpha} h^{\mu \beta} \eta_{\alpha \beta}$.

Next, we take $Y=\delta / \delta x^{\alpha}, Z=\delta / \delta x^{\mu}$, and in turn $X=$ $\delta / \delta x^{0}$, and $X=\partial / \partial x^{4}$ in (5.3), and by using (3.2), (4.4), (4.10), (5.4), (5.6(b)), and (5.6(c)), we deduce that

(a) $\Gamma_{\alpha}^{\gamma}{ }^{\gamma}=\Theta_{\alpha}^{\gamma}+\Phi^{2} \omega_{\alpha}^{\gamma}$,

(b) $\Gamma_{\alpha 4}^{\gamma}=K_{\alpha}^{\gamma}-\Psi^{2} \eta_{\alpha}^{\gamma}$.

Now, let $T$ be a spatial tensor field of type $(p, q)$. Then $\nabla_{\frac{\delta}{\delta x \beta}} T$ is a spatial tensor of type $(p, q+1)$, while $\nabla_{\frac{\delta}{\delta x} 0} T$ and $\nabla_{\frac{\partial}{\partial x^{4}}} T$ are spatial tensor fields of the same type $(p, q)$. In particular, take $T=\left(T_{\alpha}^{\gamma}\right)$ and express these three types of covariant derivatives as follows:
(a) $T_{\left.\alpha\right|_{\beta}}^{\gamma}=\frac{\delta T_{\alpha}^{\gamma}}{\delta x^{\beta}}+T_{\alpha}^{\mu} \Gamma_{\mu \beta}^{\gamma}-T_{\mu}^{\gamma} \Gamma_{\alpha \beta}^{\mu}$,
(b) $T_{\left.\alpha\right|_{0}}^{\gamma}=\frac{\delta T_{\alpha}^{\gamma}}{\delta x^{0}}+T_{\alpha}^{\mu} \Gamma_{\mu 0}^{\gamma}-T_{\mu}^{\gamma} \Gamma_{\alpha 0}^{\mu}$,
(c) $T_{\left.\alpha\right|_{4}}^{\gamma}=\frac{\partial T_{\alpha}^{\gamma}}{\partial x^{4}}+T_{\alpha}^{\mu} \Gamma_{\mu 4}^{\gamma}-T_{\mu}^{\gamma} \Gamma_{\alpha 4}^{\mu}$.

As $\nabla$ is a metric connection on $\mathcal{S} \bar{M}$, we have

(a) $h_{\left.\alpha \beta\right|_{a}}=0$, (b) $h_{\left.\right|_{a}}^{\alpha \beta}=0, \quad \forall \alpha, \beta \in\{1,2,3\}$, $a \in\{0,1,2,3,4\}$.

Finally, by using (5.3), the spatial tensor fields introduced in the previous section, and the local coefficients of the Riemannian spatial connection, we express the Levi-Civita connection of the 5D universe $(\bar{M}, \bar{g})$, as follows: 
(a) $\bar{\nabla}_{\frac{\delta}{\delta x^{\beta}}} \frac{\delta}{\delta x^{\alpha}}=\Gamma_{\alpha}^{\gamma} \beta \frac{\delta}{\delta x^{\gamma}}+\left(\omega_{\alpha \beta}+\Phi^{-2} \Theta_{\alpha \beta}\right) \frac{\delta}{\delta x^{0}}$

$+\left(\eta_{\alpha \beta}-\Psi^{-2} K_{\alpha \beta}\right) \frac{\partial}{\partial x^{4}}$,

(b) $\bar{\nabla}_{\frac{\delta}{\delta x^{0}}} \frac{\delta}{\delta x^{\alpha}}=\Gamma_{\alpha}^{\gamma} 0 \frac{\delta}{\delta x^{\gamma}}+\left(\phi_{\alpha}-b_{\alpha}\right) \frac{\delta}{\delta x^{0}}$

$+\frac{1}{2}\left(\Phi^{2} d_{\alpha} \Psi^{-2}-a_{\alpha}\right) \frac{\partial}{\partial x^{4}}$,

(c) $\bar{\nabla}_{\frac{\partial}{\partial x^{4}}} \frac{\delta}{\delta x^{\alpha}}=\Gamma_{\alpha}^{\gamma} 4 \frac{\delta}{\delta x^{\gamma}}+\frac{1}{2}\left(\Psi^{2} a_{\alpha} \Phi^{-2}-d_{\alpha}\right) \frac{\delta}{\delta x^{0}}$

$+\left(\psi_{\alpha}-c_{\alpha}\right) \frac{\partial}{\partial x^{4}}$,

(d) $\bar{\nabla}_{\frac{\delta}{\delta x^{\alpha}}} \frac{\delta}{\delta x^{0}}=\Gamma_{\alpha}{ }^{\gamma} \frac{\delta}{\delta x^{\gamma}}+\phi_{\alpha} \frac{\delta}{\delta x^{0}}+\frac{1}{2}\left(\Phi^{2} d_{\alpha} \Psi^{-2}+a_{\alpha}\right) \frac{\partial}{\partial x^{4}}$,

(e) $\bar{\nabla}_{\frac{\delta}{\delta x^{\alpha}}} \frac{\partial}{\partial x^{4}}=\Gamma_{\alpha}^{\gamma} \frac{\delta}{\delta x^{\gamma}}+\frac{1}{2}\left(\Psi^{2} a_{\alpha} \Phi^{-2}+d_{\alpha}\right) \frac{\delta}{\delta x^{0}}+\psi_{\alpha} \frac{\partial}{\partial x^{4}}$,

(f) $\bar{\nabla}_{\frac{\partial}{\partial x^{4}}} \frac{\delta}{\delta x^{0}}=\frac{1}{2}\left(\Psi^{2} a^{\gamma}-\Phi^{2} d^{\gamma}\right) \frac{\delta}{\delta x^{\gamma}}+\phi_{4} \frac{\delta}{\delta x^{0}}+\left(\psi_{0}-a_{0}\right) \frac{\partial}{\partial x^{4}}$,

(g) $\bar{\nabla}_{\frac{\delta}{\delta x^{0}}} \frac{\partial}{\partial x^{4}}=\frac{1}{2}\left(\Psi^{2} a^{\gamma}-\Phi^{2} d^{\gamma}\right) \frac{\delta}{\delta x^{\gamma}}+\phi_{4} \frac{\delta}{\delta x^{0}}+\psi_{0} \frac{\partial}{\partial x^{4}}$,

(h) $\bar{\nabla}_{\frac{\delta}{\delta x^{0}}} \frac{\delta}{\delta x^{0}}=\Phi^{2}\left(\phi^{\gamma}-b^{\gamma}\right) \frac{\delta}{\delta x^{\gamma}}+\phi_{0} \frac{\delta}{\delta x^{0}}+\Phi^{2} \phi_{4} \Psi^{-2} \frac{\partial}{\partial x^{4}}$,

(i) $\bar{\nabla} \frac{\partial}{\partial x^{4}} \frac{\partial}{\partial x^{4}}=\Psi^{2}\left(c^{\gamma}-\psi^{\gamma}\right) \frac{\delta}{\delta x^{\gamma}}+\Psi^{2}\left(\psi_{0}-a_{0}\right) \Phi^{-2} \frac{\delta}{\delta x^{0}}$

$+\psi_{4} \frac{\partial}{\partial x^{4}}$,

where we put
(a) $\phi_{0}=\Phi^{-1} \frac{\delta \Phi}{\delta x^{0}}$,
(c) $\psi_{0}=\Psi^{-1} \frac{\delta \Psi}{\delta x^{0}}$,
(b) $\phi_{4}=\Phi^{-1} \frac{\partial \Phi}{\partial x^{4}}$,
(d) $\psi_{4}=\Psi^{-1} \frac{\partial \Psi}{\partial x^{4}}$.

\section{Covariant derivatives of 4D and 5D velocities}

In this section we show that the covariant derivatives of both the $4 \mathrm{D}$ velocity $\xi^{\star}$ and the 5D velocity $\eta^{\star}$ are completely determined by the kinematic quantities and the spatial tensor fields we introduced in Sects. 4 and 5. Also, we compare the results with what is known in the $(1+3)$ threading of the 4D spacetime.

First, by using (3.5) and taking into account that $\bar{\nabla}$ is a metric connection, we obtain

(a) $\left(\bar{\nabla}_{X} \xi^{\star}\right)(Y)=\bar{g}\left(Y, \bar{\nabla}_{X} \xi\right)$,

(b) $\left(\bar{\nabla}_{X} \eta^{\star}\right)(Y)=\bar{g}\left(Y, \bar{\nabla}_{X} \eta\right), \quad \forall X, Y \in \Gamma(T \bar{M})$.

Then consider the 4D acceleration $\dot{\xi}_{a}$ and the 5D acceleration $\dot{\eta}_{a}$, given by

(a) $\dot{\xi}_{a}=\left(\bar{\nabla}_{\frac{\delta}{\delta x^{0}}} \xi^{\star}\right)\left(\frac{\partial}{\partial x^{a}}\right)$,

(b) $\dot{\eta}_{a}=\left(\bar{\nabla}_{\frac{\partial}{\partial x^{4}}} \eta^{\star}\right)\left(\frac{\partial}{\partial x^{a}}\right)$.

Next, by using (3.9) we express the natural frame field $\left\{\partial / \partial x^{a}\right\}$ in terms of the adapted frame field, as follows:

$\frac{\partial}{\partial x^{a}}=\delta_{a}^{\alpha} \frac{\delta}{\delta x^{\alpha}}-\Phi^{-2} \xi_{a} \frac{\delta}{\delta x^{0}}+\Psi^{-2} \eta_{a} \frac{\partial}{\partial x^{4}}$.
Then, by using (5.12h), (5.12i), (6.1), (6.2), and (6.3), we infer that

(a) $\dot{\xi}_{a}=\Phi^{2} \delta_{a}^{\alpha}\left(\phi_{\alpha}-b_{\alpha}\right)+\phi_{0} \xi_{a}+\phi_{4} \Phi^{2} \eta_{a} \Psi^{-2}$,

(b) $\dot{\eta}_{a}=\Psi^{2} \delta_{a}^{\alpha}\left(c_{\alpha}-\psi_{\alpha}\right)+\Psi^{2}\left(\psi_{0}-a_{0}\right) \Phi^{-2} \xi_{a}$

$+\psi_{4} \eta_{a}$

Now, by using (5.9), (5.12), (6.1) and (6.3), we obtain

(a) $\left(\bar{\nabla}_{\frac{\partial}{\partial x^{b}}} \xi^{\star}\right)\left(\frac{\delta}{\delta x^{\alpha}}\right)=\delta_{b}^{\gamma}\left(\Theta_{\alpha \gamma}+\Phi^{2} \omega_{\alpha \gamma}\right)+\xi_{b}\left(b_{\alpha}-\phi_{\alpha}\right)$ $+\frac{1}{2} \eta_{b}\left(a_{\alpha}-\Phi^{2} d_{\alpha} \Psi^{-2}\right)$,

(b) $\left(\bar{\nabla}_{\frac{\partial}{\partial x^{b}}} \xi^{\star}\right)\left(\frac{\delta}{\delta x^{0}}\right)=-\Phi^{2} \delta_{b}^{\gamma} \phi_{\gamma}+\phi_{0} \xi_{b}-\phi_{4} \Phi^{2} \eta_{b} \Psi^{-2}$,

(c) $\left(\bar{\nabla}_{\frac{\partial}{\partial x^{b}}} \xi^{\star}\right)\left(\frac{\partial}{\partial x^{4}}\right)=\frac{1}{2} \delta_{b}^{\gamma}\left(\Phi^{2} d_{\gamma}+\Psi^{2} a_{\gamma}\right)-\phi_{4} \xi_{b}$

$+\left(\psi_{0}-a_{0}\right) \eta_{b}$

and

(a) $\left(\bar{\nabla}_{\frac{\partial}{\partial x^{b}}} \eta^{\star}\right)\left(\frac{\delta}{\delta x^{\alpha}}\right)=\delta_{b}^{\gamma}\left(K_{\alpha \gamma}-\Psi^{2} \eta_{\alpha \gamma}\right)$ $+\frac{1}{2} \xi_{b}\left(d_{\alpha}-\Psi^{2} a_{\alpha} \Phi^{-2}\right)+\eta_{b}\left(c_{\alpha}-\psi_{\alpha}\right)$,

(b) $\left(\bar{\nabla}_{\frac{\partial}{\partial x^{b}}} \eta^{\star}\right)\left(\frac{\delta}{\delta x^{0}}\right)=-\frac{1}{2} \delta_{b}^{\gamma}\left(\Phi^{2} d_{\gamma}+\Psi^{2} a_{\gamma}\right)+\phi_{4} \xi_{b}$ $+\left(a_{0}-\psi_{0}\right) \eta_{b}$

(c) $\left(\bar{\nabla} \frac{\partial}{\partial x^{b}} \eta^{\star}\right)\left(\frac{\partial}{\partial x^{4}}\right)=\Psi^{2} \delta_{b}^{\gamma} \psi_{\gamma}-\psi_{0} \Psi^{2} \eta_{b} \Phi^{-2}$ $+\psi_{4} \eta_{b}$.

Finally, taking into account (6.3)-(6.6), we deduce that

$$
\begin{aligned}
\bar{\nabla}_{b} \xi_{a}= & -\Phi^{-2} \xi_{b} \dot{\xi}_{a}+\delta_{a}^{\alpha} \delta_{b}^{\beta}\left(\Theta_{\alpha \beta}+\Phi^{2} \omega_{\alpha \beta}\right)+\xi_{a} \delta_{b}^{\gamma} \phi_{\gamma} \\
& +\phi_{4} \Psi^{-2} \xi_{a} \eta_{b}+\Psi^{-2}\left(\psi_{0}-a_{0}\right) \eta_{a} \eta_{b}+\frac{1}{2} a_{\gamma}\left(\eta_{a} \delta_{b}^{\gamma}\right. \\
& \left.+\eta_{b} \delta_{a}^{\gamma}\right)+\frac{1}{2} \Phi^{2} d_{\gamma} \Psi^{-2}\left(\eta_{a} \delta_{b}^{\gamma}-\eta_{b} \delta_{a}^{\gamma}\right)
\end{aligned}
$$

and

$$
\begin{aligned}
\bar{\nabla}_{b} \eta_{a}= & \Psi^{-2} \eta_{b} \dot{\eta}_{a}+\delta_{a}^{\alpha} \delta_{b}^{\beta}\left(K_{\alpha \beta}-\Psi^{2} \eta_{\alpha \beta}\right)+\eta_{a} \delta_{b}^{\gamma} \psi_{\gamma} \\
& -\psi_{0} \Phi^{-2} \eta_{a} \xi_{b}-\phi_{4} \Phi^{-2} \xi_{a} \xi_{b}+\frac{1}{2} d_{\gamma}\left(\xi_{a} \delta_{b}^{\gamma}+\xi_{b} \delta_{a}^{\gamma}\right) \\
& +\frac{1}{2} \Psi^{2} a_{\gamma} \Phi^{-2}\left(\xi_{a} \delta_{b}^{\gamma}-\xi_{b} \delta_{a}^{\gamma}\right)
\end{aligned}
$$

Next, we note that each spatial tensor field defines a tensor field of the same type on $\bar{M}$. Here, we give some examples:

$\bar{\omega}_{a b}=\delta_{a}^{\alpha} \delta_{b}^{\beta} \omega_{\alpha \beta} ; \quad \bar{\Theta}_{a b}=\delta_{a}^{\alpha} \delta_{b}^{\beta} \Theta_{\alpha \beta} ; \bar{h}_{a b}=\delta_{a}^{\alpha} \delta_{b}^{\beta} h_{\alpha \beta} ;$

$\bar{\sigma}_{a b}=\delta_{a}^{\alpha} \delta_{b}^{\beta} \sigma_{\alpha \beta}=\bar{\Theta}_{a b}-\frac{1}{3} \Theta \bar{h}_{a b} ; \quad \bar{\phi}_{a}=\delta_{a}^{\alpha} \phi_{\alpha}$; etc.

Taking into account this transformation process of spatial tensor fields into tensor fields, we express (6.7) and (6.8) as follows: 


$$
\begin{aligned}
\bar{\nabla}_{b} \xi_{a}= & -\Phi^{-2} \xi_{b} \dot{\xi}_{a}+\bar{\sigma}_{a b}+\frac{1}{3} \Theta \bar{h}_{a b}+\Phi^{2} \bar{\omega}_{a b}+\xi_{a} \bar{\phi}_{b} \\
& +\phi_{4} \Psi^{-2} \xi_{a} \eta_{b}+\Psi^{-2}\left(\psi_{0}-a_{0}\right) \eta_{a} \eta_{b} \\
& +\frac{1}{2}\left(\eta_{a} \bar{a}_{b}+\eta_{b} \bar{a}_{a}\right)+\frac{1}{2} \Phi^{2} \Psi^{-2}\left(\eta_{a} \bar{d}_{b}-\eta_{b} \bar{d}_{a}\right)
\end{aligned}
$$

and

$$
\begin{aligned}
\bar{\nabla}_{b} \eta_{a}= & \Psi^{-2} \eta_{b} \dot{\eta}_{a}+\bar{H}_{a b}+\frac{1}{3} K \bar{h}_{a b}-\Psi^{2} \bar{\eta}_{a b}+\eta_{a} \bar{\psi}_{b} \\
& -\psi_{0} \Phi^{-2} \eta_{a} \xi_{b}-\phi_{4} \Phi^{-2} \xi_{a} \xi_{b}+\frac{1}{2}\left(\xi_{a} \bar{d}_{b}+\xi_{b} \bar{d}_{a}\right) \\
& +\frac{1}{2} \Psi^{2} \Phi^{-2}\left(\xi_{a} \bar{a}_{b}-\xi_{b} \bar{a}_{a}\right) .
\end{aligned}
$$

Now, we consider some particular cases. First, suppose that both $\xi$ and $\eta$ are unit vector fields, that is, $\Phi^{2}=\Psi^{2}=1$. Then (6.9) and (6.10) become

$$
\begin{aligned}
\bar{\nabla}_{b} \xi_{a}= & -\xi_{b} \dot{\xi}_{a}+\bar{\sigma}_{a b}+\frac{1}{3} \Theta \bar{h}_{a b}+\bar{\omega}_{a b}-a_{0} \eta_{a} \eta_{b} \\
& +\frac{1}{2}\left\{\eta_{a}\left(\bar{a}_{b}+\bar{d}_{b}\right)+\eta_{b}\left(\bar{a}_{a}-\bar{d}_{a}\right)\right\}
\end{aligned}
$$

and

$$
\begin{aligned}
\bar{\nabla}_{b} \eta_{a}= & \eta_{b} \dot{\eta}_{a}+\bar{H}_{a b}+\frac{1}{3} K \bar{h}_{a b}-\bar{\eta}_{a b} \\
& +\frac{1}{2}\left\{\xi_{a}\left(\bar{d}_{b}+\bar{a}_{b}\right)+\xi_{b}\left(\bar{d}_{a}-\bar{a}_{a}\right)\right\} .
\end{aligned}
$$

Remark 6.1 Note that the first four terms in the right hand side of (6.11) look formally as the ones in the $(1+3)$ threading of a $4 \mathrm{D}$ spacetime ([1, p. 85]). The other terms show the contribution of the fifth dimension in the kinematic theory of the $5 \mathrm{D}$ universe $(\bar{M}, \bar{g})$.

Finally, suppose that the following conditions are satisfied:

(i) The distributions $\mathcal{S} \bar{M} \oplus \mathcal{T} \bar{M}$ and $\mathcal{S} \bar{M} \oplus \mathcal{V} \bar{M}$ are integrable.

(ii) The two vector fields $\xi$ and $\eta$ define congruences of geodesics.

By (4.4) we see that condition (i) is equivalent to (4.6) and

$a_{\alpha}=d_{\alpha}=0, \quad \forall \alpha \in\{1,2,3\}$.

Also, from (5.12h), (5.12i), and (6.4), we deduce that condition (ii) is equivalent to

(a) $\phi_{\alpha}=b_{\alpha}, \phi_{0}=0, \phi_{4}=0, \dot{\xi}_{a}=0$,

(b) $\psi_{\alpha}=c_{\alpha}, \psi_{0}=a_{0}, \psi_{4}=0, \dot{\eta}_{a}=0$.

Then, by using (4.6), (6.13), and (6.14) in (6.9) and (6.10), we obtain

$\bar{\nabla}_{b} \xi_{a}=\bar{\sigma}_{a b}+\frac{1}{3} \Theta \bar{h}_{a b}+\xi_{a} \bar{\phi}_{b}$ and

$\bar{\nabla}_{b} \eta_{a}=\bar{H}_{a b}+\frac{1}{3} K \bar{h}_{a b}+\eta_{a} \bar{\psi}_{b}-\psi_{0} \Phi^{-2} \eta_{a} \xi_{b}$.

It is an interesting (and difficult) question to find solutions for the Einstein equations in a 5D universe satisfying the conditions (i) and (ii).

\section{Raychaudhuri equations in a 5D universe}

It is well known that the evolution of the expansion in a 4D spacetime is governed by the Raychaudhuri equation, which also plays an important role in the proof of Penrose-Hawking singularity theorems. So, we need to study the evolutions of both the 4D and 5D expansions $\Theta$ and $K$, given by (4.11). Such a study leads us to some equations of Raychaudhuri type, expressing the derivatives of $\Theta$ and $K$ with respect to both variables $x^{0}$ (time) and $x^{4}$ (fifth dimension). In what follows, $\bar{R}$ denotes both curvature tensor fields of the 5D universe $(\bar{M}, \bar{g})$ of types $(0,4)$ and $(1,3)$, given by

(a) $\bar{R}(X, Y, Z, U)=g(\bar{R}(X, Y, U), Z)$,

(b) $\bar{R}(X, Y, U)=\bar{\nabla}_{X} \bar{\nabla}_{Y} U-\bar{\nabla}_{Y} \bar{\nabla}_{X} U-\bar{\nabla}_{[X, Y]} U$,

for all $X, Y, Z, U \in \Gamma(T \bar{M})$. Now, we consider an orthonormal frame field $\left\{E_{k}, \Phi^{-1} \frac{\delta}{\delta x^{0}}, \Psi^{-1} \frac{\partial}{\partial x^{4}}\right\}$, where $\left\{E_{\gamma}\right\}, \gamma \in$ $\{1,2,3\}$, is an orthonormal basis in $\Gamma(\mathcal{S} \bar{M})$. Then we put

$E_{\gamma}=E_{\gamma}^{\alpha} \frac{\delta}{\delta x^{\alpha}}$

and we deduce that

$h^{\alpha \beta}=\sum_{\gamma=1}^{3} E_{\gamma}^{\alpha} E_{\gamma}^{\beta}$.

By evaluating some local components of the Ricci tensor Ric of $(\bar{M}, \bar{g})$, we shall obtain equations of Raychaudhuri type about $\Theta$ and $K$. According to ([13, p. 87]), and using (7.2) and (7.3), we obtain

$$
\begin{aligned}
\operatorname{Ric}(X, Y)= & h^{\alpha \beta} \bar{R}\left(\frac{\delta}{\delta x^{\beta}}, X, \frac{\delta}{\delta x^{\alpha}}, Y\right)-\Phi^{-2} \bar{R}\left(\frac{\delta}{\delta x^{0}}, X, \frac{\delta}{\delta x^{0}}, Y\right), \\
& +\Psi^{-2} \bar{R}\left(\frac{\partial}{\partial x^{4}}, X, \frac{\partial}{\partial x^{4}}, Y\right), \quad \forall X, Y \in \Gamma(T \bar{M}) .
\end{aligned}
$$

For our purpose, we consider only the local components:

(a) $\bar{R}_{00}=\operatorname{Ric}\left(\frac{\delta}{\delta x^{0}}, \frac{\delta}{\delta x^{0}}\right)=h^{\alpha \beta} \bar{R}\left(\frac{\delta}{\delta x^{\beta}}, \frac{\delta}{\delta x^{0}}, \frac{\delta}{\delta x^{\alpha}}, \frac{\delta}{\delta x^{0}}\right)$ $+\Psi^{-2} \bar{R}\left(\frac{\partial}{\partial x^{4}}, \frac{\delta}{\delta x^{0}}, \frac{\partial}{\partial x^{4}}, \frac{\delta}{\delta x^{0}}\right)$

(b) $\bar{R}_{44}=\operatorname{Ric}\left(\frac{\partial}{\partial x^{4}}, \frac{\partial}{\partial x^{4}}\right)=h^{\alpha \beta} \bar{R}\left(\frac{\delta}{\delta x^{\beta}}, \frac{\partial}{\partial x^{4}}, \frac{\delta}{\delta x^{\alpha}}, \frac{\partial}{\partial x^{4}}\right)$ $-\Phi^{-2} \bar{R}\left(\frac{\delta}{\delta x^{0}}, \frac{\partial}{\partial x^{4}}, \frac{\delta}{\delta x^{0}}, \frac{\partial}{\partial x^{4}}\right)$, 
(c) $\bar{R}_{04}=\overline{\operatorname{Ric}}\left(\frac{\delta}{\delta x^{0}}, \frac{\partial}{\partial x^{4}}\right)$

$$
=h^{\alpha \beta} \bar{R}\left(\frac{\delta}{\delta x^{\beta}}, \frac{\delta}{\delta x^{0}}, \frac{\delta}{\delta x^{\alpha}}, \frac{\partial}{\partial x^{4}}\right) .
$$

Next, we show the calculations for the right hand side of (7.5(a)). First, by using the symmetries of $\bar{R}$ and (7.1(a)), we obtain

$$
\begin{aligned}
& \bar{R}\left(\frac{\delta}{\delta x^{\beta}}, \frac{\delta}{\delta x^{0}}, \frac{\delta}{\delta x^{\alpha}}, \frac{\delta}{\delta x^{0}}\right)=\bar{R}\left(\frac{\delta}{\delta x^{0}}, \frac{\delta}{\delta x^{\beta}}, \frac{\delta}{\delta x^{0}}, \frac{\delta}{\delta x^{\alpha}}\right) \\
& =\bar{g}\left(\bar{R}\left(\frac{\delta}{\delta x^{0}}, \frac{\delta}{\delta x^{\beta}}, \frac{\delta}{\delta x^{\alpha}}\right), \frac{\delta}{\delta x^{0}}\right) .
\end{aligned}
$$

Then, denote by $\mathcal{T}$ the projection morphism of $\Gamma(T M)$ to $\Gamma(\mathcal{T} \bar{M})$ with respect to $(2.12)$, and by using $(4.4 \mathrm{a}),(5.12)$, (5.13(a)), and (7.1(b)), we deduce that

$$
\begin{aligned}
\mathcal{T} & \bar{R}\left(\frac{\delta}{\delta x^{0}}, \frac{\delta}{\delta x^{\beta}}, \frac{\delta}{\delta x^{\alpha}}\right)=\left\{\omega_{\left.\alpha \beta\right|_{0}}+\Phi^{-2} \Theta_{\left.\alpha \beta\right|_{0}}+\left(\omega_{\alpha \gamma}\right.\right. \\
+ & \left.\Phi^{-2} \Theta_{\alpha \gamma}\right) \Gamma_{\beta}{ }_{\beta}^{\gamma}+\phi_{0}\left(\omega_{\alpha \beta}-\Phi^{-2} \Theta_{\alpha \beta}\right)+\phi_{4}\left(\eta_{\alpha \beta}\right. \\
- & \left.\Psi^{-2} K_{\alpha \beta}\right)-\left(\phi_{\alpha}-b_{\alpha}\right)_{\left.\right|_{\beta}}-\left(\phi_{\alpha}-b_{\alpha}\right)\left(\phi_{\beta}-b_{\beta}\right) \\
& \left.+\frac{1}{4}\left(a_{\alpha}-\Phi^{2} d_{\alpha} \Psi^{-2}\right) d_{\beta}-\frac{3}{4}\left(d_{\alpha}-\Psi^{2} a_{\alpha} \Phi^{-2}\right) a_{\beta}\right\} \frac{\delta}{\delta x^{0}} .
\end{aligned}
$$

Now, we put
(a) $\omega_{\alpha \beta} \omega^{\alpha \beta}=\omega^{2}$,
(b) $\sigma_{\alpha \beta} \sigma^{\alpha \beta}=\sigma^{2}$,
(c) $\eta_{\alpha \beta} \eta^{\alpha \beta}=\eta^{2}$,
(d) $H_{\alpha \beta} H^{\alpha \beta}=H^{2}$,

and we obtain
(a) $\omega_{\gamma}^{\beta} \omega_{\beta}^{\gamma}=-\omega^{2}$,
(b) $\Theta_{\gamma}^{\beta} \Theta_{\beta}^{\gamma}=\sigma^{2}+\frac{1}{3} \Theta^{2}$,
(c) $\eta_{\gamma}^{\beta} \eta_{\beta}^{\gamma}=-\eta^{2}$
(d) $K_{\gamma}^{\beta} K_{\beta}^{\gamma}=H^{2}+\frac{1}{3} K^{2}$.

Moreover, by using the symmetries of the expansion and vorticity tensor fields, we infer that
(a) $\Theta_{\gamma}^{\beta} \omega_{\beta}^{\gamma}=0$
(b) $K_{\gamma}^{\beta} \eta_{\beta}^{\gamma}=0$

Contracting (7.6) by $h^{\alpha \beta}$ and using (2.11), (5.9(a)), (5.11(b)), (7.7), (7.9(a)), (7.9(b)), and (7.10(a)), we obtain

$$
\begin{aligned}
& h^{\alpha \beta} \bar{R}\left(\frac{\delta}{\delta x^{\beta}}, \frac{\delta}{\delta x^{0}}, \frac{\delta}{\delta x^{\alpha}}, \frac{\delta}{\delta x^{0}}\right)=-\Theta_{\left.\right|_{0}}-\sigma^{2}-\frac{1}{3} \Theta^{2} \\
& -\frac{3}{4} \Psi^{2} a^{2}+\phi_{0} \Theta+\Phi^{2}\left\{\phi_{4} \Psi^{-2} K+\left.\left(\phi^{\gamma}-b^{\gamma}\right)\right|_{\gamma}\right. \\
& \left.+\left(\phi^{\gamma}-b^{\gamma}\right)\left(\phi_{\gamma}-b_{\gamma}\right)+\frac{1}{2} a_{\gamma} d^{\gamma}\right\} \\
& +\Phi^{4}\left\{\omega^{2}+\frac{1}{4} \Psi^{-2} d^{2}\right\},
\end{aligned}
$$

where we put

$\Theta_{\left.\right|_{0}}=\frac{\delta \Theta}{\delta x^{0}}, \quad a^{2}=a_{\gamma} a^{\gamma}, \quad d^{2}=d_{\gamma} d^{\gamma}$.

Similar calculations as performed for (7.7) lead to the following:

$$
\begin{aligned}
\mathcal{T} & \bar{R}\left(\frac{\delta}{\delta x^{0}}, \frac{\partial}{\partial x^{4}}, \frac{\partial}{\partial x^{4}}\right)=\left\{\Psi^{2}\left(c^{\gamma}-\psi^{\gamma}\right)\left(\phi_{\gamma}-b_{\gamma}\right)\right. \\
& -\frac{1}{4} \Psi^{4} a^{2} \Phi^{-2}-\frac{1}{4} \Phi^{2} d^{2}-\left(\phi_{4}\right)^{2}-\frac{\partial \phi_{4}}{\partial x^{4}} \\
& +\frac{1}{2} \Psi^{2} a_{\gamma} d^{\gamma}+\phi_{4} \psi_{4}+\left[\Psi^{2}\left(\psi_{0}-a_{0}\right) \Phi^{-2}\right]_{\left.\right|_{0}} \\
& \left.+\Psi^{2}\left(\psi_{0}-a_{0}\right)\left(\phi_{0}-\psi_{0}-a_{0}\right) \Phi^{-2}\right\} \frac{\delta}{\delta x^{0}}
\end{aligned}
$$

Then, by using (2.11), (7.1(a)), and (7.12), we deduce that

$$
\begin{aligned}
\bar{R} & \left(\frac{\partial}{\partial x^{4}}, \frac{\delta}{\delta x^{0}}, \frac{\partial}{\partial x^{4}}, \frac{\delta}{\delta x^{0}}\right) \\
= & \Phi^{2} \Psi^{2}\left\{\left(\phi^{\gamma}-b^{\gamma}\right)\left(\psi_{\gamma}-c_{\gamma}\right)-\frac{1}{2} a_{\gamma} d^{\gamma}\right\} \\
& +\Phi^{2}\left\{\left(\phi_{4}\right)^{2}+\frac{\partial \phi_{4}}{\partial x^{4}}-\phi_{4} \psi_{4}\right\}+\Psi^{2}\left\{( \psi _ { 0 } - a _ { 0 } ) \left(\phi_{0}\right.\right. \\
& \left.\left.-\psi_{0}+a_{0}\right)-\left(\psi_{0}-a_{0}\right)_{\left.\right|_{0}}\right\}+\frac{1}{4}\left\{\Phi^{4} d^{2}+\Psi^{4} a^{2}\right\} .
\end{aligned}
$$

Finally, by using (7.11) and (7.13) in (7.5(a)), we infer that

$$
\begin{aligned}
& \Theta_{\left.\right|_{0}}=-\sigma^{2}-\frac{1}{3} \Theta^{2}-\frac{1}{2} \Psi^{2} a^{2}+\phi_{0} \Theta-\left(\psi_{0}-a_{0}\right)_{\left.\right|_{0}} \\
& +\left(\psi_{0}-a_{0}\right)\left(\phi_{0}-\psi_{0}+a_{0}\right)+\Phi^{2}\left\{\phi_{4} \Psi^{-2} K+\left(\phi^{\gamma}-b^{\gamma}\right)_{\left.\right|_{\gamma}}\right. \\
& +\left(\phi_{\gamma}-b_{\gamma}\right)\left(\phi^{\gamma}-b^{\gamma}-c^{\gamma}+\psi^{\gamma}\right)+\Phi^{2}\left(\omega^{2}+\frac{1}{2} \Psi^{-2} d^{2}\right) \\
& \left.+\Psi^{-2}\left(\left(\phi_{4}\right)^{2}+\frac{\partial \phi_{4}}{\partial x^{4}}-\phi_{4} \psi_{4}\right)\right\}-\bar{R}_{00} .
\end{aligned}
$$

We call (7.14) the 4D Raychaudhuri equation for the 4D expansion $\Theta$ of the 5D universe $(\bar{M}, \bar{g})$.

By similar calculations, we obtain

$$
\begin{aligned}
\bar{R} & \left(\frac{\delta}{\delta x^{\beta}}, \frac{\partial}{\partial x^{4}}, \frac{\delta}{\delta x^{\alpha}}, \frac{\partial}{\partial x^{4}}\right)=\bar{g}\left(\bar{R}\left(\frac{\partial}{\partial x^{4}}, \frac{\delta}{\delta x^{\beta}}, \frac{\delta}{\delta x^{\alpha}}\right), \frac{\partial}{\partial x^{4}}\right) \\
= & \Psi^{2}\left\{\eta_{\alpha \beta \mid 4}-\Psi^{-2} K_{\alpha \beta \mid 4}-\left(\psi_{\alpha}-c_{\alpha}\right)_{\mid \beta}\right. \\
& +\left(\eta_{\alpha \gamma}-\Psi^{-2} K_{\alpha \gamma}\right) \Gamma_{\beta 4}^{\gamma} \\
& +\left(\omega_{\alpha \beta}+\Phi^{-2} \Theta_{\alpha \beta}\right)\left(\psi_{0}-a_{0}\right) \\
& +\left(\eta_{\alpha \beta}+\Psi^{-2} K_{\alpha \beta}\right) \psi_{4}+\frac{3}{4}\left(\Phi^{2} d_{\alpha} \Psi^{-2}-a_{\alpha}\right) d_{\beta} \\
& \left.+\frac{1}{4}\left(d_{\alpha}-\Psi^{2} a_{\alpha} \Phi^{-2}\right) a_{\beta}-\left(\psi_{\alpha}-c_{\alpha}\right)\left(\psi_{\beta}-c_{\beta}\right)\right\} .
\end{aligned}
$$


Then, by using (7.13) and (7.15) in (7.5b), we deduce that

$$
\begin{aligned}
K_{\mid 4} & =-H^{2}-\frac{1}{3} K^{2}+\Psi^{4} \eta^{2}-\Psi^{2}\left\{\left(\psi^{\gamma}-c^{\gamma}\right)_{\mid \gamma}\right. \\
& \left.+\left(\psi^{\gamma}-c^{\gamma}\right)\left(\phi_{\gamma}-b_{\gamma}+\psi_{\gamma}-c_{\gamma}\right)\right\} \\
& +\Psi^{2} \Phi^{-2}\left\{\left(\psi_{0}-a_{0}\right)_{\mid 0}+\left(\psi_{0}-a_{0}\right)\left(\psi_{0}-\phi_{0}\right.\right. \\
& \left.\left.-a_{0}+\Theta\right)-\frac{1}{2} \Psi^{2} a^{2}\right\}+\phi_{4} K+\frac{1}{2} \Phi^{2} d^{2}-\left(\phi_{4}\right)^{2} \\
& -\frac{\partial \phi_{4}}{\partial x^{4}}+\phi_{4} \psi_{4}-\bar{R}_{44},
\end{aligned}
$$

where we put

$$
K_{\mid 4}=\frac{\partial K}{\partial x^{4}} .
$$

We call (7.16) the 5D Raychaudhuri equation for the 5D expansion $K$ of the 5D universe $(\bar{M}, \bar{g})$.

Finally, we shall state some Raychaudhuri equations which involve

$\Theta_{\mid 4}=\frac{\partial \Theta}{\partial x^{4}}$, and $K_{\mid 0}=\frac{\delta K}{\delta x^{0}}$.

First, by using (2.3), (4.4a), (5.12), and (7.1), we infer that

$$
\begin{aligned}
\bar{R} & \left(\frac{\delta}{\delta x^{\beta}}, \frac{\delta}{\delta x^{0}}, \frac{\delta}{\delta x^{\alpha}}, \frac{\partial}{\partial x^{4}}\right)=\bar{R}\left(\frac{\delta}{\delta x^{0}}, \frac{\delta}{\delta x^{\beta}}, \frac{\partial}{\partial x^{4}}, \frac{\delta}{\delta x^{\alpha}}\right) \\
= & \bar{g}\left(\bar{R}\left(\frac{\delta}{\delta x^{0}}, \frac{\delta}{\delta x^{\beta}}, \frac{\delta}{\delta x^{\alpha}}\right), \frac{\partial}{\partial x^{4}}\right) \\
= & \Psi^{2} \eta_{\alpha \beta \mid 0}-K_{\alpha \beta \mid 0}+\frac{1}{2}\left(a_{\alpha}-\Phi^{2} d_{\alpha} \Psi^{-2}\right)_{\mid \beta} \Psi^{2} \\
& +\left(\Psi^{2} \eta_{\alpha \gamma}-K_{\alpha \gamma}\right) \Gamma_{\beta 0}^{\gamma}+\Psi^{2}\left(\psi_{\alpha}-c_{\alpha}\right) a_{\beta} \\
& +\left(\Psi^{2} \eta_{\alpha \beta}+K_{\alpha \beta}\right) \psi_{0}-\frac{1}{2}\left(\phi_{\alpha}-b_{\alpha}\right)\left(\Phi^{2} d_{\beta}+\Psi^{2} a_{\beta}\right) \\
& -\frac{1}{2}\left(\Phi^{2} d_{\alpha}-\Psi^{2} a_{\alpha}\right)\left(\psi_{\beta}-b_{\beta}\right) \\
& +\left(\Phi^{2} \omega_{\alpha \beta}+\theta_{\alpha \beta}\right) \phi_{4} .
\end{aligned}
$$

Contracting (7.17) by $h^{\alpha \beta}$, and using (4.11), (5.9a), (5.11b), and (7.5c), we obtain

$$
\begin{aligned}
K_{\mid 0}= & \frac{1}{2}\left(a^{\gamma}-\Phi^{2} d^{\gamma} \Psi^{-2}\right)_{\mid \gamma} \Psi^{2}-K_{\alpha \beta} \Theta^{\alpha \beta}+\psi_{0} K \\
& +\phi_{4} \Theta-\frac{1}{2} \Phi^{2} d_{\gamma}\left(\phi^{\gamma}+\psi^{\gamma}-2 b^{\gamma}\right)+\Psi^{2}\left\{\frac { 1 } { 2 } a _ { \gamma } \left(3 \psi^{\gamma}\right.\right. \\
& \left.\left.-\phi^{\gamma}-2 c^{\gamma}\right)-\Phi^{2} \eta_{\alpha \beta} \omega^{\alpha \beta}\right\}-\bar{R}_{04} .
\end{aligned}
$$

On the other hand, we calculate $\bar{R}$ from (7.5c) by using (2.11), (4.4(b)), (5.12), and (7.1) to deduce that

$$
\begin{aligned}
\bar{R} & \left(\frac{\delta}{\delta x^{\beta}}, \frac{\delta}{\delta x^{0}}, \frac{\delta}{\delta x^{\alpha}}, \frac{\partial}{\partial x^{4}}\right) \\
= & \bar{R}\left(\frac{\partial}{\partial x^{4}}, \frac{\delta}{\delta x^{\alpha}}, \frac{\delta}{\delta x^{0}}, \frac{\delta}{\delta x^{\beta}}\right) \\
= & \bar{g}\left(\bar{R}\left(\frac{\partial}{\partial x^{4}}, \frac{\delta}{\delta x^{\alpha}}, \frac{\delta}{\delta x^{\beta}}\right), \frac{\delta}{\delta x^{0}}\right) \\
= & -\Theta_{\alpha \beta \mid 4}+\Phi^{2} \omega_{\alpha \beta \mid 4} \\
& -\frac{1}{2}\left(d_{\beta}-\Psi^{2} a_{\beta} \Phi^{-2}\right)_{\mid \alpha} \Phi^{2}+\left(\Phi^{2} \omega_{\gamma \beta}-\theta_{\gamma \beta}\right) \Gamma_{\alpha 4}^{\gamma} \\
& +\left(\Phi^{2} \omega_{\alpha \beta}+\theta_{\alpha \beta}\right) \phi_{4} \\
& +\Psi^{2}\left(\psi_{0}-a_{0}\right)\left(\eta_{\alpha \beta}+\Psi^{-2} K_{\alpha \beta}\right) \\
& +\frac{1}{2} \phi_{\alpha}\left(\Psi^{2} a_{\beta}-\Phi^{2} d_{\beta}\right)+\frac{1}{2}\left(\Psi^{2} a_{\alpha}+\Phi^{2} d_{\alpha}\right) \\
& \times\left(\psi_{\beta}-c_{\beta}\right)+\Phi^{2} d_{\alpha}\left(b_{\beta}-\phi_{\beta}\right) \\
& +\frac{1}{2} c_{\alpha}\left(\Phi^{2} d_{\beta}-\Psi^{2} a_{\beta}\right) .
\end{aligned}
$$

Then, by using (7.19) in (7.5(c)), and taking into account (4.11), (5.9(b)), and (5.11(b)), we infer that

$$
\begin{aligned}
\Theta_{\mid 4}= & \frac{1}{2}\left(\Psi^{2} a^{\gamma} \Phi^{-2}-d^{\gamma}\right)_{\mid \gamma} \Phi^{2}-K_{\alpha \beta} \Theta^{\alpha \beta}+\left(\psi_{0}-a_{0}\right) K \\
& +\phi_{4} \Theta-\frac{1}{2} \Phi^{2} d_{\gamma}\left(3 \phi^{\gamma}-\psi^{\gamma}-2 b^{\gamma}\right) \\
& +\Psi^{2}\left\{\frac{1}{2} a_{\gamma}\left(\phi^{\gamma}+\psi^{\gamma}-2 c^{\gamma}\right)-\Phi^{2} \eta_{\alpha \beta} \omega^{\alpha \beta}\right\} \\
& -\bar{R}_{04} .
\end{aligned}
$$

By elementary calculations using (4.7), we obtain

(a) $\left(\Psi^{2} a^{\gamma} \Phi^{-2}-d^{\gamma}\right)_{\mid \gamma} \Phi^{2}=\left(\Psi^{2} a^{\gamma}-\Phi^{2} d^{\gamma}\right)_{\mid \gamma}$ $+2 \phi_{\gamma}\left(\Phi^{2} d^{\gamma}-\Psi^{2} a^{\gamma}\right)$

(b) $\left(a^{\gamma}-\Phi^{2} d^{\gamma} \Psi^{-2}\right)_{\mid \gamma} \Psi^{2}=\left(\Psi^{2} a^{\gamma}-\Phi^{2} d^{\gamma}\right)_{\mid \gamma}$

$+2 \psi_{\gamma}\left(\Phi^{2} d^{\gamma}-\Psi^{2} a^{\gamma}\right)$.

Finally, by using (7.21(a)) in (7.20), and (7.21(b)) in (7.18), we deduce that

$$
\begin{aligned}
\Theta_{\mid 4}= & K_{\mid 0}-a_{0} K=\frac{1}{2}\left(\Psi^{2} a^{\gamma}-\Phi^{2} d^{\gamma}\right)_{\mid \gamma}-K_{\alpha \beta} \Theta^{\alpha \beta} \\
& -\Phi^{2} \Psi^{2} \eta_{\alpha \beta} \omega^{\alpha \beta}+\left(\psi_{0}-a_{0}\right) K+\phi_{4} \Theta+\frac{1}{2} \Phi^{2}\left(\Psi^{\gamma}\right. \\
& \left.-\phi^{\gamma}+2 b^{\gamma}\right) d_{\gamma}+\frac{1}{2} \Psi^{2}\left(\psi^{\gamma}-\phi^{\gamma}-2 c^{\gamma}\right) a_{\gamma} \\
& -\bar{R}_{04} .
\end{aligned}
$$

We call (7.22) the mixed Raychaudhuri equation in the 5D universe $(\bar{M}, \bar{g})$.

The classical Raychaudhuri equation in a 4D spacetime has been generalized in several directions. We recall here three such generalizations. First, in [14] there were 
stated Raychaudhuri equations for one single and two nonnormalized vector fields in a 4D spacetime. Our Raychaudhuri equations are also with respect to two non-normalized vector fields, but in a 5D universe. Then in [15] there have been obtained generalizations of the Raychaudhuri equation for the evolution of deformations of a relativistic membrane of arbitrary dimension in an arbitrary background spacetime. The approach we developed in this paper is totally different from the one presented in [15]. Also, the Raychaudhuri equation and singularity theorems have been recently stated in a Finsler spacetime (cf. [16]).

Note that the above Raychaudhuri equations have been obtained in the most general 5D universe. In particular, suppose that the lift of $\partial / \partial x^{0}$ from the $4 \mathrm{D}$ spacetime to the $5 \mathrm{D}$ universe is orthogonal to $\partial / \partial x^{4}$. Then by $(2.9(\mathrm{~b}))$ and (2.10) we deduce that $A_{0}=0$, which implies

$\delta / \delta x^{0}=\partial / \partial x^{0}$,

via (2.7). Now, each point $\mathrm{P}$ of $\bar{M}$ can be considered as intersection point of an integral curve of $\partial / \partial x^{0}$ on which we choose $x^{0}$ as parameter, with an integral curve of $\partial / \partial x^{4}$ parametrized by $x^{4}$. Since in this case $a_{0}=0$ [see (3.1(b))], from (7.22) and (7.23) we deduce that

$$
\frac{\mathrm{d} \Theta}{\mathrm{d} x^{4}}=\frac{\mathrm{d} K}{\mathrm{~d} x^{0}},
$$

at any point $P \in \bar{M}$. Thus, the rate of change of the 4D expansion $\Theta$ in the direction of the fifth dimension, is equal to the rate of change of the $5 \mathrm{D}$ expansion $K$ in the time direction.

Finally, we prove the following important results for the kinematic theory of a 5D universe.

Theorem 7.1 Let $(\bar{M}, \bar{g})$ be a $5 D$ universe satisfying the following conditions:

(a) The distribution $\mathcal{S} \bar{M} \oplus \mathcal{V} \bar{M}$ is integrable.

(b) $\xi=\delta / \delta x^{0}$ is given by (7.23) and defines a congruence of geodesics.

(c) $\bar{R}_{00} \geq 0, \psi_{0 \mid 0} \geq 0$ on $\bar{M}$.

If the $4 D$ expansion $\Theta$ takes the negative value $\Theta_{0}$ at a point of a geodesic in the congruence, then $\Theta$ goes to $-\infty$ along that geodesic and the proper time coordinate $x^{0}$ satisfies $x^{0} \leq-3 / \Theta_{0}$.

Proof Taking into account (4.4(b)) and (4.4(c)), the condition (a) is equivalent to

$d_{\alpha}=0$ and $\omega_{\alpha \beta}=0, \quad \forall \alpha, \beta \in\{1,2,3\}$.

Then note that condition (b) implies $A_{0}=0$ and (6.14(a)). Taking into account that $a_{0}=0$ [cf. (3.1(b))], and using (6.14(a)) and (7.25) in the 4D Raychaudhuri equation (7.14), we obtain

$\Theta_{\mid 0}=-\sigma^{2}-\frac{1}{3} \Theta^{2}-\frac{1}{2} \Psi^{2} a^{2}-\left(\psi_{0}\right)^{2}-\psi_{0 \mid 0}-\bar{R}_{00}$.

Finally, by condition (c) we see that (7.26) implies

$\Theta_{\mid 0}+\frac{1}{3} \Theta^{2} \leq 0$

along any geodesic $C$ that is tangent to $\xi$. Choose $x^{0}$ as a parameter on $C$ and by (7.23), we express (7.27) as follows:

$\frac{\mathrm{d} \Theta}{\mathrm{d} x^{0}}+\frac{1}{3} \Theta^{2}\left(x^{0}\right) \leq 0$,

along $C$. By using (7.28), and for the same reason as in the kinematic theory of a 4D spacetime (see the proof of Lemma 9.2.1 in [17]), we obtain the assertion of the theorem.

Remark 7.1 The conditions (7.23) and $\psi_{0 \mid 0} \geq 0$ are new conditions compared with a similar result in a 4D spacetime. We note that (7.23) is equivalent to $\bar{g}_{04}=0$, which is satisfied by all the $5 \mathrm{D}$ models from both the brane-world theory $[8,9]$ and the space-time-matter theory $[10,11]$. Also, $\psi_{0 \mid 0}=0$ for any 5D universe in which $\eta$ is a unit vector field.

Theorem 7.2 Let $(\bar{M}, \bar{g})$ be a $5 D$ universe satisfying the following conditions:

(a) The distributions $\mathcal{S} \bar{M} \oplus \mathcal{V} \bar{M}$ and $\mathcal{S} \bar{M} \oplus \mathcal{T} \bar{M}$ are integrable.

(b) The two vector fields $\xi$ and $\eta$ define congruences of geodesics.

(c) $\bar{R}_{44} \geq 0$, on $\bar{M}$.

If the $5 D$ expansion $K$ takes the negative value $K_{0}$ at a point of a geodesic determined by $\eta$, then $K$ goes to $-\infty$ along that geodesic and the fifth coordinate $x^{4}$ satisfies $x^{4} \leq-3 / K_{0}$.

Proof The condition (a) is equivalent to (7.25) and

$a_{\alpha}=0$ and $\eta_{\alpha \beta}=0, \quad \forall \alpha, \beta \in\{1,2,3\}$,

via (4.4(a)) and (4.4(c)). Also, note that condition (b) is equivalent to (6.14). Then, by using (6.14), (7.25), and (7.29) in the 5D Raychaudhuri equation (7.16), we obtain

$K_{\left.\right|_{4}}=-H^{2}-\frac{1}{3} K^{2}-\bar{R}_{44}$.

The condition (c) and (7.30) imply

$K_{\mid 4}+\frac{1}{3} K^{2} \leq 0$,

on any geodesic $C$ defined by $\eta$. Consider $x^{4}$ as a parameter on $C$ and by using (7.31) and for the same reason as in Theorem 7.1, we obtain the assertion of the theorem. 
Theorems 7.1 and 7.2 might be useful in an attempt to prove singularity theorems for a 5D universe. Also, the three types of Raychaudhuri equations (7.14), (7.16), and (7.22) might have an important role in a study of the evolution of a concrete 5D universe.

\section{Conclusions}

In the present paper, for the first time in the literature, we develop a kinematic theory in a 5D universe. This can be considered as an extension of the $(1+3)$ threading of spacetime with respect to an arbitrary timelike vector field that has been developed in [18]. The main tools in our approach are the spatial tensor fields and the Riemannian connection defined on the spatial distribution. It is worth mentioning that all the kinematic quantities (acceleration, expansion, shear and vorticity) are defined as spatial tensor fields, and therefore they should be considered as 3D geometric objects in a 5D universe.

Now, we stress on the novelty brought about by our paper for the study of a 5D universe. First, we mention the $(1+1+3)$ splitting determined by the two vector fields $\xi$ and $\eta$, which has an important role in relating geometry and physics to the observations. In this way, the 5D universe is filled up by nets determined by integral curves of both $\xi$ and $\eta$. So, apart from the 4D kinematic quantities related to $\xi$, should be taken into consideration those determined by $\eta$, provided the fifth dimension does exist in our universe. Also, it is important to mention the three types of Raychaudhuri equations, which we obtained in the $5 \mathrm{D}$ universe $(\bar{M}, \bar{g})$. They describe the evolution of both the 4D expansion and the 5D expansion along the two congruences determined by $\xi$ and $\eta$. Theorems 7.1 and 7.2 prove the existence of the singularities in both expansions $\Theta$ and $K$. Actually, these theorems state that caustics will develop in both congruences if a convergence occurs anywhere. Taking into account the methods used to prove the Penrose-Hawking singularity theorems in a 4D spacetime (cf. [19]), we think that such theorems might play an important role in a proof of the existence of singularities in a 5D universe. Finally, we should mention that our study is mainly developed on the mathematical part of the kinematic theory in a 5D universe. This must be followed by detailed studies which might bring new insights on the 4D physics in the presence of the fifth dimension.

Open Access This article is distributed under the terms of the Creative Commons Attribution 4.0 International License (http://creativecomm ons.org/licenses/by/4.0/), which permits unrestricted use, distribution, and reproduction in any medium, provided you give appropriate credit to the original author(s) and the source, provide a link to the Creative Commons license, and indicate if changes were made.

Funded by SCOAP $^{3}$.

\section{References}

1. G.F.R. Ellis, R. Maartens, M.A.H. MacCallum, Relativistic Cosmology (Cambridge University Press, Cambridge, 2012)

2. D. Bini, P. Carini, R.T. Jantzen, Class. Quantum Gravity 12, 2549 (1995)

3. R. Maartents, B.A. Basset, Class. Quantum Gravity 15, 705 (1998)

4. B. Mashhoon, J.C. McClune, H. Quevedo, Class. Quantum Gravity 16, 1137 (1999)

5. G.F.R. Ellis, H. van Elst, R. Maartens, Class. Quantum Gravity 18, 5115 (2001)

6. S.W. Hawking, Astrophys. J. 145, 544 (1966)

7. R. Maartens, Phys. Rev. D 55, 463 (1997)

8. R. Maartens, K. Koyama, Living Rev. Relativ. 13, 5 (2010)

9. D. Langlois, Prog. Theor. Phys. Suppl. 148, 181 (2002)

10. J.M. Overduin, P.S. Wesson, Phys. Rep. 283, 303 (1999)

11. P.S. Wesson, Space-Time-Matter. Modern Kaluza-Klein Theory (World Scientific, Singapore, 1999), p. 1997

12. A. Bejancu, H.R. Farran, Foliations and Geometric Structures (Springer, Dordrecht, 2006)

13. B. O’Neill, Semi-Riemannian Geometry and Applications to Relativity (Academic Press, New York, 1983)

14. G. Abreu, M. Visser, Phys. Rev. D 83, 104016 (2011)

15. R. Capovilla, J. Guven, Phys. Rev. D 52, 1072 (1995)

16. E. Minguzzi, Raychaudhuri equation and singularity theorems in Finsler spaces. arXiv:1502.02313 [gr-qc] (2015)

17. R.M. Wald, General Relativity (The University of Chicago Press, Chicago, 1984)

18. A. Bejancu, C. Calin, Eur. Phys. J. C 75, 159 (2015)

19. S.W. Hawking, G.F.R. Ellis, The Large Scale Structure of SpaceTime (Cambridge University Press, Cambridge, 1973) 